\title{
First-principles Fourier approach for the calculation of the effective dielectric constant of periodic composites
}

\author{
Ruibao Tao, ${ }^{*}$ Zhe Chen, ${ }^{\dagger}$ and Ping Sheng \\ Exxon Research \& Engineering Co., Route 22 East Clinton Township, Annandale, New Jersey 08801
}

(Received 11 July 1989; revised manuscript received 22 September 1989)

\begin{abstract}
We present a simple first-principles Fourier approach to the calculation of the effective dielectric constant of periodic composites. The method involves the solution of the inhomogeneous Maxwell equations, in which the spatially dependent dielectric constant $\epsilon(\mathbf{r})$ is represented by its Fourier components and the resulting field equations are solved in the wave-vector domain. This approach avoids the matching of boundary conditions along the material interfaces inherent in the traditional boundary-value approach, thus offering generality and simplicity for handling periodic systems with arbitrary unit-cell geometries. Application of the approach is illustrated by two examples, and the results are compared with known solutions.
\end{abstract}

For inhomogeneous materials, the fact that there is always a unique effective dielectric constant in the static limit may be viewed as resulting from the inability of the probing wave to resolve the individual inhomogeneities when the wavelength is much larger than the intrinsic correlation length of the system. The material would thus appear homogeneous to the probing wave with an effective dielectric constant whose value is a function of the dielectric constants of the constituent phases. In recent years, it has been increasingly realized that, although the low-frequency waves cannot "see" the inhomogeneities, the value of the effective dielectric constant is nevertheless critically dependent on the microgeometry of the composite, i.e., the shape and topological arrangements of the components. ${ }^{1}$ To take this microstructural effect into account theoretically would require the solution of boundary-value problems defined on domains with arbitrary geometries. While special shapes such as circle, sphere, square, and cube are associated with well-defined basis functions and would therefore be amenable to attack in the manner of the traditional boundary-value matching approach, the very efficiency and accuracy gained in specializing to a particular geometry generally make such methods difficult to accommodate the mixture of geometries encountered even in simple periodic composites.

In this article, we present a first-principles Fourier approach to the calculations of the effective dielectric constant for periodic composites that avoids the difficult task of boundary-condition matching along material interfaces that is inherent in the traditional approach. ${ }^{2}$ The method involves the solution of inhomogeneous Maxwell equations in which the dielectric constant $\epsilon(\mathbf{r})$ is represented in terms of its Fourier components, and the resulting field equations are solved in the wave-vector domain. The accuracy of the approach is tested by the calculation of two examples whose solutions are known.

Consider the inhomogeneous wave equation that can be directly obtained from Maxwell equations:

$$
-\frac{\mu \epsilon(\mathbf{r}) \omega^{2}}{c^{2}} \mathbf{E}(\mathbf{r})=\nabla^{2} \mathbf{E}(\mathbf{r})-\nabla[\nabla \cdot \mathbf{E}(\mathbf{r})],
$$

where $\mu$ is the magnetic permeability, assumed to be a constant, $c$ denotes the speed of light, $\omega$ is the angular frequency, and we have assumed $\mathbf{E}(\mathbf{r}, t)=\mathbf{E}(\mathbf{r}) \exp (-i \omega t)$. In Eq. (1), $\boldsymbol{\nabla} \cdot \mathbf{E}(\mathbf{r}) \neq 0$ because

$$
\boldsymbol{\nabla} \cdot[\epsilon(\mathbf{r}) \mathbf{E}(\mathbf{r})]=0 \text {. }
$$

Fourier transform of Eqs. (1) and (2) gives

$$
\begin{aligned}
& \left(\frac{\mu \epsilon(0) \omega^{2}}{c^{2}}-k^{2}\right) \mathbf{E}(\mathbf{k})+\mathbf{k}[\mathbf{k} \cdot \mathbf{E}(\mathbf{k})] \\
& =-\frac{\mu \omega^{2}}{c^{2}} \sum_{\mathbf{G}_{n} \neq 0} \epsilon\left(\mathbf{G}_{n}\right) \mathbf{E}\left(\mathbf{k}-\mathbf{G}_{n}\right), \\
& \mathbf{k} \cdot \sum_{\mathbf{G}_{n}} \epsilon\left(\mathbf{G}_{n}\right) \mathbf{E}\left(\mathbf{k}-\mathbf{G}_{n}\right)=0 \text {. }
\end{aligned}
$$

Here $\epsilon\left(\mathbf{G}_{n}\right)$ and $\mathbf{E}(\mathbf{k})$ are the Fourier components of $\epsilon(\mathbf{r})$ and $\mathbf{E}(\mathbf{r})$, respectively, and $\mathbf{G}_{n}$ denotes the reciprocal lattice vector of the periodic structure. We will denote the solution to Eqs. (3) and (4) in the limit of $|\mathbf{k}| \rightarrow 0$ as $\mathbf{E}^{(0)}(\mathbf{k})$ and $\mathbf{E}^{(0)}\left(\mathbf{k}-\mathbf{G}_{n}\right)$. The requirement that at $\mathbf{k} \rightarrow 0$ the composite material should appear homogeneous means that

$$
\mathbf{k} \cdot \mathbf{E}^{(0)}(\mathbf{k})=0
$$

so that $\mathbf{E}^{(0)}(\mathbf{k})$ can represent the transverse electric field of an electromagnetic wave in a homogeneous medium. That implies $\mathbf{E}^{(0)}(\mathbf{k})$ has only two independent vector components.

In order to calculate the effective dielectric constant of $\bar{\epsilon}$ of the composite, we need to derive the matrix $\overleftrightarrow{\mathrm{S}}^{ \pm}$that relates $\mathbf{E}^{(0)}(\mathbf{k})$ to $\mathbf{E}^{(0)}\left(\mathbf{k} \pm \mathbf{G}_{n}\right)$ :

$$
\mathbf{E}^{(0)}\left(\mathbf{k} \pm \mathbf{G}_{n}\right)=\stackrel{\mathbf{S}^{ \pm}}{ }\left(\mathbf{G}_{n}\right) \cdot \mathbf{E}^{(0)}(\mathbf{k}) \text {. }
$$

By denoting the unit vector in the direction of 
$\mathbf{E}^{(0)}\left(\mathbf{k} \mp \mathbf{G}_{n}\right)$ as $\pm \hat{\mathbf{g}}_{n}$ and that in the direction of $\mathbf{E}^{(0)}(\mathbf{k})$ as $\hat{\mathbf{e}}$, we can express $\overleftrightarrow{\mathbf{S}}^{ \pm}(\mathbf{G})$ alternatively as

$$
\overleftrightarrow{\mathbf{S}}^{ \pm}\left(\mathbf{G}_{n}\right)=S^{ \pm}\left(\mathbf{G}_{n}\right)\left[\hat{\mathbf{g}}_{n} \otimes \hat{\mathbf{e}}\right],
$$

where the symbol $\otimes$ denotes the outer product between two vectors. If the quantities $S^{ \pm}\left(G_{n}\right)$ are known, then the substitution of Eqs. (5), (6), and (7) into Eq. (3) gives

$$
\left[\frac{\mu \epsilon(0) \omega^{2}}{c^{2}}(1+A)-k^{2}\right) E^{(0)}(\mathbf{k})=0,
$$

where

$$
A=\sum_{\mathbf{G}_{n} \neq 0} \frac{\epsilon\left(\mathbf{G}_{n}\right)}{\epsilon(0)} S^{-}\left(\mathbf{G}_{n}\right)\left(\hat{\mathbf{g}}_{n} \cdot \hat{\mathbf{e}}\right)
$$

Equation (8a) defines the dispersion relation of a wave in the effective medium with a dielectric constant

$$
\bar{\epsilon}=\epsilon(0)(1+A) .
$$

The knowledge of $S^{-}\left(G_{n}\right)$ is therefore seen to directly yield the effective dielectric constant. To get $S^{-}\left(\mathbf{G}_{n}\right)$, we return to Eq. (3) and make the substitution of $\mathbf{k}-\mathbf{G}_{m}$ for $\mathbf{k}$. In the limit of $|\mathbf{k}| \rightarrow 0$, the resulting leading order terms give

$$
G_{m}^{2} \mathbf{E}^{(0)}\left(\mathbf{k}-\mathbf{G}_{m}\right)=\mathbf{G}_{m}\left[\mathbf{G}_{m} \cdot \mathbf{E}^{(0)}\left(\mathbf{k}-\mathbf{G}_{m}\right)\right],
$$

which essentially defines the direction of $\mathbf{E}^{(0)}\left(\mathbf{k}-\mathbf{G}_{n}\right)$ as being parallel to $\mathbf{G}_{n}$. In other words, $\hat{\mathbf{g}}_{n}=\mathbf{G} /\left|\mathbf{G}_{n}\right|$. Similar substitution in Eq. (4) and the evaluation in the $|\mathbf{k}| \rightarrow 0$ limit yields

$$
\mathbf{G}_{m}\left(\epsilon(0) \mathbf{E}^{(0)}\left(\mathbf{k}-\mathbf{G}_{m}\right)+\epsilon(-\mathbf{G}) \mathbf{E}^{(0)}(\mathbf{k})+\sum_{\substack{\mathbf{G}_{n} \neq 0 \\ \neq \mathbf{G}_{m}}} \epsilon\left(\mathbf{G}_{n}-\mathbf{G}_{m}\right) \mathbf{E}^{(0)}\left(\mathbf{k}-\mathbf{G}_{n}\right)\right]=0
$$

By using Eq. (10a), Eq. (10b) may be reexpressed as

$$
E^{(0)}\left(\mathbf{k}-\mathbf{G}_{m}\right)=-\frac{\epsilon\left(\mathbf{G}_{m}\right)}{\epsilon(0)}\left(\hat{\mathbf{g}}_{m} \cdot \hat{\mathbf{e}}\right) E^{(0)}(\mathbf{k})-\sum_{\substack{\mathbf{G}_{n} \neq 0 \\ \neq \mathbf{G}_{m}}} \frac{\epsilon\left(\mathbf{G}_{n}-\mathbf{G}_{m}\right)}{\epsilon(0)}\left(\hat{\mathbf{g}}_{m} \cdot \hat{\mathbf{g}}_{n}\right) E^{(0)}\left(\mathbf{k}-\mathbf{G}_{n}\right) .
$$

Comparison with Eqs. (6) and (7) then directly gives

$$
S^{-}\left(\mathbf{G}_{m}\right)=-\frac{\epsilon\left(-\mathbf{G}_{m}\right)}{\epsilon(0)}\left(\hat{\mathbf{g}}_{m} \cdot \hat{\mathbf{e}}\right)-\sum_{\substack{\mathbf{G}_{n} \neq 0 \\ \neq \mathbf{G}_{m}}} \frac{\epsilon\left(\mathbf{G}_{n}-\mathbf{G}_{m}\right)}{\epsilon(0)}\left(\hat{\mathbf{g}}_{m} \cdot \hat{\mathbf{g}}_{n}\right) S^{-}\left(\mathbf{G}_{n}\right) .
$$

Equation (12) represents an infinite set of coupled linear simultaneous equations whose solution is the central task of the present Fourier approach. The inputs to the equations are seen to be just $\epsilon\left(\mathbf{G}_{n}\right)$ and the unit reciprocal lattice vectors of the structure. In the following we illustrate the application of this Fourier approach by two examples, one two dimensional (2D) and the other three dimensional (3D), and compare the results with known solutions.

The geometries of the two periodic composites are shown in Figs. 1(a) and 1(b). For the first case, we have a two-dimensional composite whose unit-cell geometry is shown in Fig. 1(a). The unit cell consists of a square diamond of $\epsilon_{1}$ and half-diagonal $\eta$ inside a square of $\epsilon_{2}$ and side 2 . The reciprocal lattice vectors are given by

$$
\mathbf{G}_{n}=\pi\left(n_{x}, n_{y}\right), \quad n_{x}, n_{y}=0, \pm 1, \pm 2, \ldots,
$$

and the Fourier component of $\epsilon(r)$ may be expressed as

$$
\begin{aligned}
\epsilon\left(\mathbf{G}_{n}\right)= & \left(\epsilon_{1}-\epsilon_{2}\right) \frac{\cos G_{n y} \eta-\cos G_{n x} \eta}{G_{n x}^{2}-G_{n y}^{2}} \\
& +\epsilon_{2} \frac{\sin G_{n x} \sin G_{n y}}{G_{n x} G_{n y}} .
\end{aligned}
$$

The area fraction of component 1 is given by $f=\eta^{2} / 2$. Since we have to truncate the infinite set of simultaneous equations to within $\left|n_{x}\right|,\left|n_{y}\right|<N$, the results of the calculation at various $N$ values are extrapolated to $N=\infty$ by using the form

$$
y=A+\frac{B}{(2 N+1)^{\alpha}},
$$

where the parameters $A, B$, and $\alpha$ are obtained by nonlinear least-squares fits to calculated data. In Fig. 2 we show such extrapolations of $\epsilon_{1} / \epsilon_{2}=5$ at various area fractions of component 1 . The largest value of $N$ used in our calculation is 10 , corresponding to the solution of a $441 \times 441$ set of linear simultaneous equations. The results are tabulated in Table I for $\epsilon_{1} / \epsilon_{2}=2$ and 5 with the corresponding solution values obtained by Milton et al. ${ }^{3}$ using the boundary-value approach. It is seen that the agreement is excellent in general, with the deviation from the known results slightly larger for the $\epsilon_{1} / \epsilon_{2}=5$ case.

We can also compare our results with that of the Maxwell-Garnett (MG) theory, ${ }^{4}$ in which $\epsilon$ solves the following effective-medium equation:

$$
\frac{\epsilon-\epsilon_{2}}{\epsilon+L \epsilon_{2}}=f \frac{\epsilon_{1}-\epsilon_{2}}{\epsilon_{1}+L \epsilon_{2}} .
$$




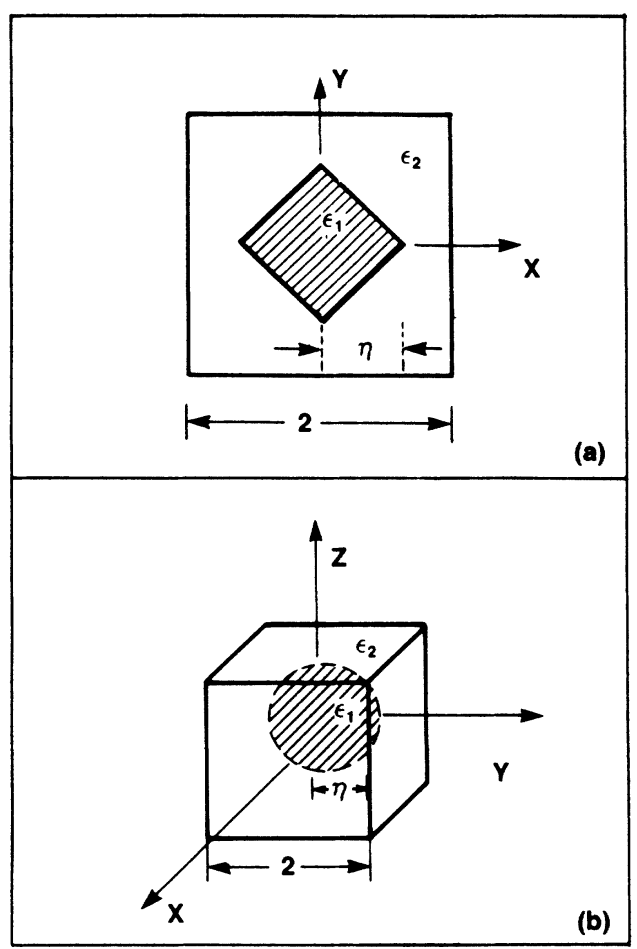

FIG. 1. (a) Geometry of the unit cell in the 2D case. The area fraction of material $\epsilon_{1}$ is $\eta^{2} / 2$. (b) Geometry of the unit cell in the 3D case. The volume fraction of material $\epsilon_{1}$ is $(\pi / 6) \eta^{3}$.

Here $L=1$ for two-dimensional systems and $L=2$ for three-dimensional systems. This is done for the $\epsilon_{1} / \epsilon_{2}=5$ case. It is seen from Table $I$ that whereas the agreement is good for $f<0.3$, the difference soon becomes significant as $f$ approaches 0.5 .

In the second example, we consider a threedimensional periodic composite whose unit-cell structure consists of a sphere of dielectric constant $\epsilon_{1}$ inscribed inside a cube with side 2 as shown in Fig. 1(b). The dielectric constant outside the sphere is denoted as $\epsilon_{2}$. The reciprocal lattice vector is given by

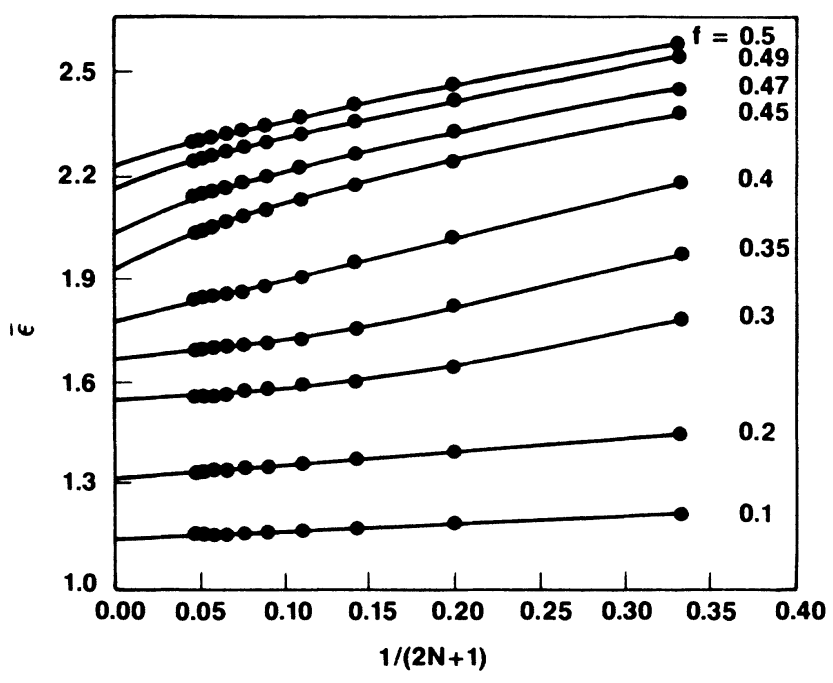

FIG. 2. Extrapolations of the effective dielectric constant for the $2 \mathrm{D}$ case. Here $\epsilon_{1} / \epsilon_{2}=5$. The values of the nonlinear extrapolation exponent $\alpha$ obtained are as follows: $0.984(f=0.1)$, $1.03(f=0.2), 1.67(f=0.3), 1.47(f=0.35), 1.04(f=0.4), 0.71$ $(f=0.45), 0.72(f=0.47), 0.8(f=0.49)$, and $0.85(f=0.5)$.

$\mathbf{G}_{n}=\pi\left(n_{x}, n_{y}, n_{z}\right) ; \quad n_{x}, n_{y}, n_{z}=0, \pm 1, \pm 2, \ldots$.

The Fourier component of $\epsilon(\mathbf{r})$ may be written as

$\epsilon\left(\mathbf{G}_{n}\right)=\frac{\pi}{2|\mathbf{G}|^{3}}\left(\epsilon_{1}-\epsilon_{2}\right)\left(\sin G_{n} \eta-G_{n} \eta \cos G_{n} \eta\right)$,

and the volume fraction of component 1 is given by $f=\pi \eta^{3} / 6$. For $\epsilon_{1} / \epsilon_{2}=3$, we have carried out calculations up to $\left|n_{x}\right|,\left|n_{y}\right|,\left|n_{z}\right|<N=4$, corresponding to the solution of a $729 \times 729$ set of linear simultaneous equations. The calculated data are again extrapolated to $N=\infty$ by using the form of Eq. (15). The final results are tabulated in Table II together with the solution values by McPhedran et al., ${ }^{5}$ obtained through the boundarymatching approach. It is seen that the agreement is generally good up to the neighborhood of $f=\pi / 6$, where the spheres touch. At $f=\pi / 6$ the deviation is about $2.5 \%$.

TABLE I. Comparison of our results with the solutions of Milton et al. (Ref. 3) for the 2D case.

\begin{tabular}{|c|c|c|c|c|c|}
\hline \multirow{2}{*}{$\underbrace{\epsilon_{1} / \epsilon_{2}}$} & \multicolumn{2}{|c|}{2} & \multicolumn{3}{|c|}{5} \\
\hline & $\begin{array}{l}\text { Milton } \\
\text { (Ref. 3) }\end{array}$ & Fourier & $\begin{array}{l}\text { Milton et al. } \\
\text { (Ref. 3) }\end{array}$ & Fourier & MG \\
\hline 0.1 & 1.0696 & 1.0695 & 1.1490 & 1.1487 & 1.1429 \\
\hline 0.2 & 1.1445 & 1.1445 & 1.3239 & 1.3233 & 1.3077 \\
\hline 0.3 & 1.2255 & 1.2247 & 1.5359 & 1.5553 & 1.5000 \\
\hline 0.35 & 1.2687 & 1.2671 & 1.6620 & 1.6752 & 1.6087 \\
\hline 0.4 & 1.3141 & 1.3120 & 1.8079 & 1.7853 & 1.7273 \\
\hline 0.43 & 1.3622 & 1.3608 & 1.9845 & 1.9138 & 1.8037 \\
\hline 0.47 & 1.3824 & 1.3816 & 2.0694 & 2.0224 & 1.9126 \\
\hline 0.48 & 1.3927 & 1.3924 & 2.117 & 2.0962 & 1.9412 \\
\hline 0.49 & 1.4032 & 1.4032 & 2.170 & 2.1651 & 1.9703 \\
\hline 0.50 & 1.4142 & 1.4141 & 2.236 & 2.2327 & 2.0000 \\
\hline
\end{tabular}


TABLE II. Comparison of our results with the solution of McPhedran et al. (Ref. 4) for the 3D case with $\epsilon_{1} / \epsilon_{2}=3$.

\begin{tabular}{|c|c|c|c|}
\hline$f$ & $\begin{array}{c}\text { McPhedran et al. } \\
\text { (Ref. 4) }\end{array}$ & Fourier & MG \\
\hline 0.1 & 1.125 & 1.126 & 1.125 \\
\hline 0.2 & 1.261 & 1.258 & 1.261 \\
\hline 0.3 & 1.410 & 1.404 & 1.409 \\
\hline 0.35 & 1.490 & 1.489 & 1.488 \\
\hline 0.4 & 1.574 & 1.585 & 1.571 \\
\hline 0.43 & 1.626 & 1.646 & 1.623 \\
\hline 0.46 & 1.680 & 1.710 & 1.676 \\
\hline 0.48 & 1.718 & 1.754 & 1.713 \\
\hline 0.5 & 1.757 & 1.797 & 1.750 \\
\hline 0.51 & 1.776 & 1.819 & 1.769 \\
\hline 0.52 & 1.796 & 1.841 & 1.788 \\
\hline 0.672 & & 2.175 & 2.103 \\
\hline 0.738 & & 2.334 & 2.257 \\
\hline 0.798 & & 2.479 & 2.406 \\
\hline 0.896 & & 2.732 & 2.676 \\
\hline
\end{tabular}

Considering that the solution accuracy is most difficult to obtain at the touching geometry, such deviation in our approach may be considered reasonable indeed.

We have also extended our calculation to $f$ values beyond the percolation threshold of $f=\pi / 6 \approx 0.53$. Here the Fourier coefficients of $\epsilon(r)$ can still be easily evaluated. ${ }^{2}$ Our results at all concentrations are also compared with the MG theory, which gives fairly reasonable results as well. Since the MG theory is based on the consideration of spherical and circular (2D) inclusions, its good agreement with the 3D model but not with the 2D model, where the inclusion is square in shape, illustrates the importance of shape in determining the effective dielectric constant. However, it should be noted that the geometric basis upon which the MG theory is derived (particles dispersed in a matrix) no longer corresponds to the actual geometry for $f>\pi / 6$. Therefore the agreement beyond $f>\pi / 6$ is fortuitous for the MG theory.

In both examples above, the Fourier approach requires only changes in the input Fourier coefficients $\epsilon\left(\mathbf{G}_{n}\right)$ when we go beyond the $f$ values where the component 1 becomes connected. However, for the second example when $f$ becomes larger than $\pi / 6$ the boundary-matching approach would immediately encounter the difficult task of matching boundary conditions on a tortuous material interface defined by fused spheres. It is therefore clear that the Fourier approach offers a significant advantage in this regard. On the negative side, the Fourier approach requires extrapolations that can be inaccurate as the ratio $\epsilon_{1} / \epsilon_{2}$ becomes large. Of course, such inaccuracies may be minimized by increasing the cutoff $N$ values. Yet the rapidly escalating size of the resulting calculation, especially in the three-dimensional case, may make such a strategy impractical.

In summary, we have formulated a Fourier approach to the calculation of the effective dielectric constant of periodic composites that can handle arbitrary unit-cell geometries. Comparisons with known calculations have shown that the method is simple and accurate when the ratio of the dielectric constants is not too large.
*Permanent address: Department of Physics, Fudan University, Shanghai, People's Republic of China.

†Also at the Department of Physics of the City College of New York, New York, NY 10031.

${ }^{1} \mathrm{P}$. Sheng, in Homogenization and Effective Moduli of Materials and Media, edited by J. L. Ericksen, D. Kinderlehrer, R. Kohn, and J. L. Kons (Springer-Verlag, New York, 1986), p. 196.

${ }^{2}$ For the effective elastic moduli of periodic composites, a similar approach has been formulated by $R$. Tao and P. Sheng. See R. Tao and P. Sheng, J. Acoust. Soc. Am. 77, 1651 (1985);
P. Sheng and R. Tao, Phys. Rev. B 31, 6131 (1985).

${ }^{3}$ G. N. Milton, R. C. McPhedran, and D. R. McKenzie, Appl. Phys. 25, 23 (1981).

${ }^{4}$ See R. Landauer, in Electrical Transport and Optical Properties of Inhomogeneous Media (Ohio State University, 1977), Proceedings of the First Conference on the Electrical Transport and Optical Properties of Inhomogeneous Media, AIP Conf. Proc. No. 40, edited J. C. Garland and D. B. Tanner (AIP, New York, 1978), p. 1.

${ }^{5}$ R. C. McPhendran and D. R. McKenzie, Proc. R. Soc. London, Ser. A 359, 45 (1978). 